\title{
IMPLICACIÓN DE LA RESILIENCIA Y DE LAS FUNCIONES EJECUTIVAS EN EL RENDIMIENTO ACADEMICO DE EDUCACIÓN OBLIGATORIA
}

\author{
Marta Bestué Laguna \\ Facultad Ciencias Humanas y de la Educación. Universidad de Zaragoza mbestue@unizar.es \\ Elena Escolano-Pérez \\ Facultad de Educación. Universidad de Zaragoza. eescola@unizar.es
}

Recepción Artículo: 27 octubre 2021 Admisión Evaluación: 27 octubre 2021 Informe Evaluador 1: 28 octubre 2021 Informe Evaluador 2: 29 octubre 2021 Aprobación Publicación: 30 octubre 2021

\section{RESUMEN}

El rendimiento académico se puede definir como el conjunto de destrezas y capacidades del alumnado para demostrar sus conocimientos sobre diferentes áreas. Actualmente, es uno de los motivos de preocupación e interés para todos los gobiernos ya que se encuentra relacionado con el desarrollo educativo y económico de un país. Especialmente, a raíz de la crisis sanitaria, se ha puesto especial interés en la capacidad de adaptación del alumnado para afrontar nuevas situaciones, resiliencia. Según otros estudios existe relación entre la resiliencia y el rendimiento académico y entre las funciones ejecutivas y este mismo. Para este estudio el objetivo fue analizar en qué medida cada una de estas variables, además del género, podían explicar la variable dependiente: rendimiento académico. Participaron 177 adolescentes de los cursos desde $1^{0}$ hasta $4^{0}$ de ESO, de los cuales el $55,4 \%$ eran hombres. Todos ellos eran alumnos de un mismo centro educativo. Para la evaluación de los problemas en el funcionamiento ejecutivo se utilizó el BRIEF-2. Para evaluar la capacidad de resiliencia se utilizó el cuestionario Connor-Davidson. Por último, para medir el rendimiento académico se tuvo en cuenta la media de las notas de las asignaturas: lengua, matemáticas, inglés, educación física y geografía e historia. Las tres variables (género, funcionamiento ejecutivo y resiliencia) quedaron incluidas en el modelo que resultó significativo con un valor explicativo del rendimiento académico de $\left(\mathrm{R}^{2}=.276 ; \mathrm{p}<.05\right)$. El género fue la variable que más contribuyó al rendimiento académico ( $\beta=.54)$, seguido de los problemas en el funcionamiento ejecutivo $(\beta=-.33)$ y la resiliencia $(\beta=.20)$. Estos resultados abordan la importancia de desarrollar programas de desarrollo del funcionamiento ejecutivo y de la resiliencia en adolescentes, especialmente del género masculino, para la mejora del rendimiento académico. Estas competencias son de gran importancia en el desempeño de funciones no solo académicas, sino de habilidades para la vida.

Palabras clave: rendimiento académico; resiliencia; ámbito educativo; funciones ejecutivas 


\title{
IMPLICACIÓN DE LA RESILIENCIA Y DE LAS FUNCIONES EJECUTIVAS EN EL RENDIMIENTO ACADÉMICO DE EDUCACIÓN OBLIGATORIA
}

\begin{abstract}
Implication of resilience and executive functions on academic performance in compulsory

education. Academic performance can be defined as the set of student s skills and abilities to demonstrate their knowledge in different areas. Currently, it is one of the reasons of concern and interest for all governments since it is related to the educational and economic development of a country. Especially, as a result of the health crisis, special interest has been placed on the adaptability of students to face new situations, resilience. According to other studies, there is a relationship between resilience and academic performance and between executive functions and academic performance. For this study, the objective was to analyze to what extent each of these variables, in addition to gender, could explain the dependent variable: academic performance. 177 adolescents participated in the courses from 1 st to 4 th of ESO, of which $55.4 \%$ were men. All of them were students from the same educational center. For the evaluation of problems in executive functioning, the BRIEF-2 was used. To assess resilience capacity, the Connor- Davidson questionnaire was used. Finally, to measure academic performance, the average of the grades for the subjects was taken into account: language, mathematics, English, physical education, and geography and history. The three variables (gender, executive functioning and resilience) were included in the model, which was significant with an explanatory value of academic performance of $\left(R^{2}=.276 ; p<.05\right)$. Gender was the variable that contributed the most to academic performance $(\beta=.54)$, followed by problems in executive functioning ( $\beta=-.33$ ) and resilience $(\beta=.20)$. These results address the importance of developing executive functioning and resilience development programs in adolescents, especially males, to improve academic performance. These competencies are of great importance in the performance of not only academic functions, but also life skills.
\end{abstract}

Keywords: academic performance; resilience; educational field; executive function

\section{INTRODUCCIÓN}

La resiliencia es una de las variables más estudiadas en la actualidad debido a la importancia que ha tenido en la salud física y mental después de la pandemia mundial derivada de la COVID-19. La resiliencia se define como uno de los factores protectores que ayudan a las personas a adaptarse ante acontecimientos adversos de gran impacto, es decir, que les permite permanecer en un nivel de funcionamiento óptimo en ese periodo de adaptación a una nueva situación adversa. (Vinaccia, Quiceno y Moreno, 2007 y Fínez-Silva, Morán-Astorga, y Urchaga-Litago, 2019)

La resiliencia y el rendimiento académico están relacionados, según estudios como el de Sánchez-Álvarez, Berrios y Extremera (2020) existe una relación entre estas variables, asumiendo que emociones negativas se consideran factores de riesgo mientras que la resiliencia, junto a otros componentes de gestión emocional, determina un buen rendimiento académico. El rendimiento académico es considerado en ocasiones como sinónimo de las notas académicas ya que estas suelen ser reflejo de una evaluación de los aprendizajes (Miñano y Castejón, 2011).

El rendimiento académico ha sido y es una de las variables más estudiadas en el entorno educativo por su gran impacto en el desarrollo tanto económico como social de un país. En educación secundaria obligatoria toma especial relevancia porque es uno de los momentos en los que se da la mayor parte del abandono escolar. Este abandono escolar puede traer consecuencias negativas no solo para el desarrollo de un país sino también para los individuos que permanecen sin una formación básica obligatoria.

Además de la resiliencia otra de las variables que se ha estudiado que tiene relación con el rendimiento académico son las funciones ejecutivas (Zelazo y Carlson, 2020).

Actualmente no existe un consenso en cuanto a la conceptualización de las funciones ejecutivas (FE), sin embargo se puede definir como el conjunto de procesos mentales que permiten a las personas comportarse de una forma flexible y adaptativa en contextos cambiantes para alcanzar una meta. Aunando definiciones como la de Lezak (1982), Sastre-Riba (2006), Miyake y Friedman (2012), Diamond (2013) y Escolano- Pérez y Bestué (2021) las funciones ejecutivas abarcan desde procesos cognitivos como la memoria de trabajo, la inhibición, la 
planificación, la flexibilidad, hasta procesos emocionales y, la coordinación de todos ellos debe ir dirigida para conseguir un objetivo. Por ello, en muchas ocasiones, la definición de las funciones ejecutivas se asemeja a un "director de orquesta" que coordina los procesos mentales del lóbulo frontal (Goldberg, 2009).

Pero ¿hay relación entre la resiliencia y las funciones ejecutivas en los adolescentes con el rendimiento académico? Según estudios como el de Zhang, Y., Zhang, X., Zhang, L. y Guo (2019) la resiliencia y las funciones ejecutivas tienen un impacto sobre el rendimiento académico donde actúan como variables mediadoras. Según Wu et al. (2021) las funciones ejecutivas y la resiliencia son los componentes clave de la capacidad de un individuo para participar de forma significativa y eficaz en su entorno. En este estudio se parte de la necesidad de explicar el rendimiento académico a través de variables que puedan verse mejoradas a través de una intervención partiendo de la plasticidad cerebral. Tanto la resiliencia como las funciones ejecutivas son factores que pueden mejorarse a través de un entrenamiento. Este tipo de intervenciones cada vez están más próximas al ámbito educativo, desde el Ministerio de Educación y Formación Profesional (2021), en su página web invitan a los docentes de educación primaria a realizar intervenciones para la mejora de las funciones ejecutivas y para el desarrollo de competencias emocionales o resiliencia. En educación secundaria obligatoria, es un periodo crítico en el que también se deben plantear este tipo de intervenciones, pero antes debemos conocer si tendrían impacto sobre el rendimiento académico.

Además, para este estudio se ha tenido en cuenta el género de los estudiantes puesto que en un amplio rango de investigaciones previas se ha demostrado que es una variable que influye tanto en el rendimiento académico (Matthews, Ponitz, y Morrison, 2009) como en la resiliencia (Sun y Stewart, 2007) y en las funciones ejecutivas (Grissom y Reyes, 2019).

\section{OBJETIVOS DE LA INVESTIGACIÓN}

El objetivo primer objetivo de este estudio es conocer si se da relación entre el rendimiento académico y las variables que hipotéticamente lo explican.

El segundo objetivo es conocer en qué medida las variables: resiliencia, funciones ejecutivas y género pueden llegar a explicar el rendimiento académico para poder plantear soluciones con una intervención neuroeducativa eficaz.

\section{MUESTRA Y/O PARTICIPANTES}

La muestra está compuesta por 177 estudiantes, de los cuales el 55,4\% (98) eran hombres y el 44,6\% (79) eran mujeres. Las edades estaban comprendidas entre los 12 y los 16 años puesto que se trata de alumnos desde $1^{0}$ hasta $4^{0}$ de educación secundaria obligatoria (ESO). Todos los participantes son estudiantes de un mismo centro educativo de España.

\section{METODOLOGÍA Y/O INSTRUMENTOS UTILIZADOS}

El instrumento utilizado para medir la resiliencia ha sido la escala de resiliencia de Connor y Davidson (2003) validada para población española de adolescentes (García, González-Gómez, Robles-Ortega, Padilla, y Peralta-Ramirez, 2019). Esta escala está compuesta por 25 ítems, las respuestas son de tipo Likert de 1 a 5, donde las puntuaciones más altas indican mayor resiliencia. Ha sido utilizada tanto en población general como en el ámbito clínico, en este caso se ha utilizado para población general. Los resultados arrojan valores tanto de la resiliencia en general como de 5 factores que

la componen: 1- persistencia, tenacidad y autoeficacia, 2- control bajo presión, 3- adaptabilidad y redes de apoyo, 4- control y propósito 5- espiritualidad. Algunos ejemplos de las preguntas de estos factores son las siguientes en cuanto al 1- Siempre me esfuerzo sin importar el resultado. Del 2- Bajo presión, me mantengo enfocado. 3- Tengo al menos una relación segura que me ayuda cuando estoy estresado. 4- Me siento en control de mi vida. 5- Buenas o malas, las cosas ocurren por alguna razón.

Para medir las funciones ejecutivas se ha utilizado el BRIEF-2 versión escuela. Es un cuestionario de refe- 
rencia a nivel mundial para evaluar las funciones ejecutivas. Este instrumento los resultados que arrojan son las dificultades en el funcionamiento ejecutivo, lo que habrá que tenerlo en consideración de cara al análisis de resultados. Este instrumento está compuesto por 63 ítems que dan información sobre las escalas de inhibición, supervisión de sí mismo, flexibilidad, control emocional, iniciativa, memoria de trabajo, planificación, supervisión de la tarea y organización de materiales. La versión escuela quiere decir que el cuestionario ha sido administrado a los docentes del centro educativo que tenían una relación estrecha con el alumnado y con la supervisión de la orientadora del centro. El índice que se ha utilizado para este estudio ha sido el índice global de la función ejecutiva. Esta batería también se responde siguiendo las respuestas de escala tipo Likert. (Gioia et al., 2017)

En cuanto al rendimiento académico se utilizaron la media de las puntuaciones académicas de las asignaturas comunes a todos los cursos desde $1^{0}$ de ESO hasta $4^{\circ}$ siendo estas: Lengua castellana y literatura, Matemáticas, Inglés, Geografía e historia y Educación física. Como ya se ha explicado en la introducción, las notas son uno de los instrumentos más utilizados para medir el rendimiento académico en estudiantes.

Para el análisis de datos se utilizó el paquete estadístico SPSS versión 27.

\section{RESULTADOS ALCANZADOS}

Para abordar el primer objetivo del estudio, el primer análisis de resultados que se realizó fueron las correlaciones que se daban entre las variables de estudio. Para seleccionar el tipo de coeficiente a utilizar, en primer lugar se analizó si la muestra seguía una distribución normal. En este caso, como no seguía estrictamente una distribución normal se seleccionó el coeficiente Rho de Spearman para analizar las correlaciones entre las variables. Los resultados obtenidos fueron los siguientes:

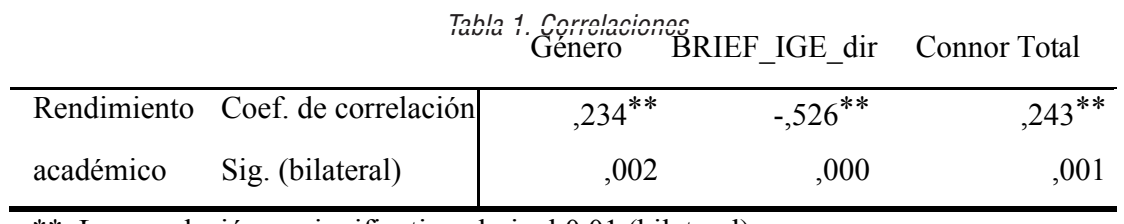

**. La correlación es significativa al nivel 0,01 (bilateral).

Siendo BRIEF_IGE_dir la puntuación directa de las dificultades en el funcionamiento ejecutivo y Connor total la puntuación directa de la variable resiliencia.

Una vez observada la relación significativa con la variable de estudio a través de las correlaciones, se acepta el supuesto de linealidad. Para dar respuesta al segundo objetivo se pasó a realizar un modelo de regresión lineal múltiple. Para ello, se deben cumplir los siguientes supuestos: la independencia de los errores, según el test de Durbin Watson siendo de 2,187 $(>1,5 \mathrm{y}<2,5)$. Otro de los supuestos es que no haya una colinealidad entre las variables independientes (funciones ejecutivas, género y resiliencia) obteniendo un valor de inflación de la varianza inferior a 10 , siendo todos los valores inferiores a 0,006 , por lo que no hay colinealidad. En cuanto al supuesto de la homocedasticidad los residuos estás distribuidos de forma adecuada. En los siguientes gráficos se pueden observar los supuestos explicados: 
Figura 1. Gráfico P-P normal de regresión Residuo estandarizado.

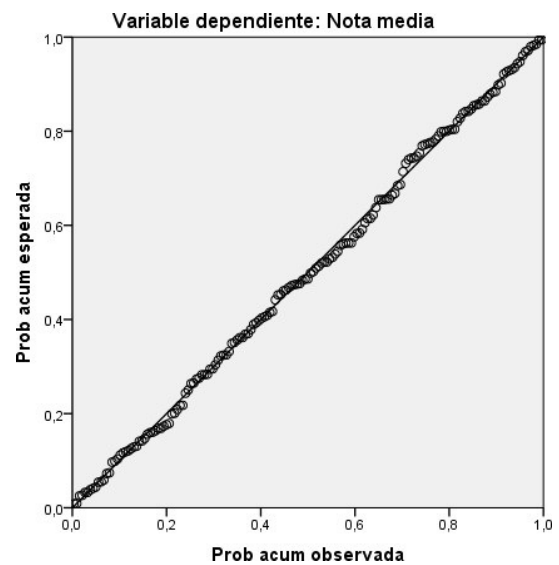

Figura 2. Gráfico histograma.

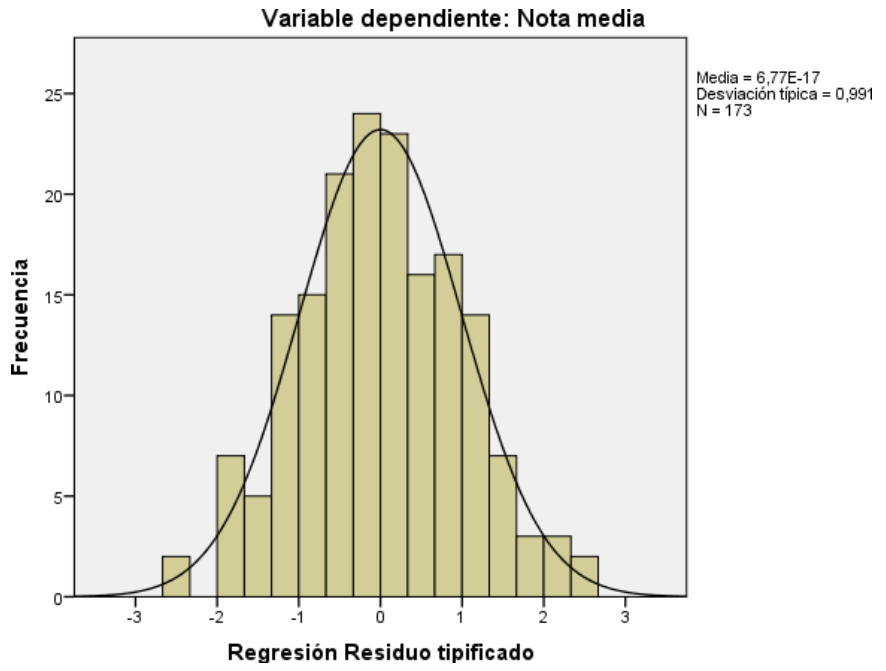

En esta última gráfica, en cuanto a la normalidad de los residuos encontramos una pequeña asimetría positiva, lo que puede suponer alguna interferencia a la hora de interpretar los datos aunque, al tener resultados que provienen de puntuaciones de escalas tipo Likert, es bastante frecuente encontrar asimetrías.

Dando respuesta al segundo objetivo encontramos un modelo regresión lineal múltiple con un $\mathrm{R}^{2}=, 276$. 
Tabla 2. Regresión lineal múltiple.
$\mathrm{R}$
R cuadrado
Error típ. de la
Durbin-Watson
estimación

\begin{tabular}{cccc}
\hline, $525 \mathrm{a}$ &, 276 & 1,263 & 2,187 \\
\hline
\end{tabular}

a. Variables predictoras: (Constante), BRIEF_IGE_dir, Connor Total, Género

b. Variable dependiente: Nota media

Además de los resultados observados en la Tabla 2, las variables predictoras del rendimiento académico son todas las variables independientes introducidas en el modelo ya que $p<.005$.

La línea de regresión de este modelo quedaría reflejada de la siguiente manera:

Rendimiento académico $=7,286+0,54$ género $+0,02$ resiliencia $-0,033$ funciones ejecutivas.

El rendimiento académico se puede predecir siguiendo la línea de regresión presentada donde influye que si se tiene buen rendimiento de las funciones ejecutivas, el sujeto es mujer y tiene alta puntuación en resiliencia, mejora el rendimiento académico.

\section{DISCUSIÓN}

A partir de los resultados encontrados podemos decir que las variables independientes del estudio (funcionamiento ejecutivo, resiliencia y género) son indicadores de predicción de la variable dependiente rendimiento académico tal como se partía del planteamiento hipotético y siguiendo los resultados de estudios previos abordados en el apartado de introducción. En este estudio las variables independientes seleccionadas llegan a explicar en un 27,6\% los resultados académicos de los estudiantes de educación secundaria obligatoria.

En este modelo de regresión la resiliencia tiene menor peso explicativo, pero es una variable que aporta de forma significativa valor a la variable rendimiento académico. En el caso del funcionamiento ejecutivo, los resultados aparecen en negativo debido a que, como ya se nombra en el apartado de instrumentos, la batería del BRIEF nos indica dificultades en el funcionamiento ejecutivo. El funcionamiento ejecutivo también en una variable que aporta valor explicativo de forma significativa al rendimiento

académico. El género es la variable que mayor peso explicativo tiene sobre la variable dependiente, por lo que se considera necesario tenerlo en cuenta de cara a futuras investigaciones e intervenciones.

En cuanto a las correlaciones, cabe destacar la dirección de esta relación, en el caso del género quiere decir que las mujeres tienen relación con un alto rendimiento académico, en el caso de la resiliencia a mayor resiliencia, mayor rendimiento y en cambio, en cuanto al funcionamiento ejecutivo es una relación inversa donde las puntuaciones altas en el mismo están relacionadas con un peor rendimiento académico.

\section{CONCLUSIONES}

El rendimiento académico es uno de los aspectos educativos más estudiados a lo largo de la historia. Es foco de multitud de normativas, Leyes, Decretos, Órdenes, que pretenden disminuir el fracaso académico y proponen actuaciones de intervención. Sin embargo, el fracaso escolar y el abandono escolar sigue estando por encima de Ios niveles deseados en España, tanto que es uno de los Objetivos de Desarrollo Sostenible (ODS) sobre los que se debe actuar según la Agenda 2030 (OECD, 2020).

Existen múltiples variables relacionadas con el rendimiento académico, pero el objetivo de este estudio era determinar aquellas que pueden ser modificables a través de una intervención o de un programa de mejora de las mismas para mejorar este rendimiento académico (Antonio-Aguirre, Rodríguez-Fernández y Revuelta, 2019). Además, en educación secundaria obligatoria es uno de los momentos de mayor sensibilidad en cuanto al desarrollo neuromadurativo y que tiene un impacto directo sobre el funcionamiento ejecutivo (Lezak, Howieson y 
Loring, 2004), por lo que en estas edades de periodo sensible es de vital importancia abordar aspectos tanto cognitivos como emocionales que faciliten la mejora del rendimiento académico.

Actualmente en España existen diversos programas educativos que favorecen el aprendizaje y el rendimiento de los estudiantes, sin embargo, están alejados de la realidad investigadora actual en donde hay mayores avances de los que quedan reflejados en la normativa. A través de los resultados de este estudio, se pretende dar a conocer la importancia de abordar tanto el desarrollo de las funciones ejecutivas como de la resiliencia teniendo en cuenta las diferencias de género, que pueden llegar a tener un efecto significativo en el rendimiento académico de los estudiantes.

La resiliencia como tal no es un factor que pueda dar respuesta al rendimiento académico, pero trabajándolo junto al desarrollo de las funciones ejecutivas y bajo la diferencia de género configuran un modelo explicativo del rendimiento académico de gran importancia. Resultados similares se encontraron en estudios que abordaban la resiliencia y el rendimiento como el de Vinaccia et al. (2007) 0 en Wu et al. (2021).

A la hora de generalizar estos resultados debemos tener en cuenta que el rendimiento académico en este estudio se ha valorado teniendo en cuenta la nota media de un grueso

de asignaturas y que podrían haberse medido a través de otros instrumentos (Miñano y Castejón, 2011), sin embargo, actualmente, son los resultados de esas notas medias los que determinan un fracaso escolar 0 actuaciones generales como las repeticiones, por lo que se considera un buen factor de medición teniendo en cuenta la normativa actual (LOMLOE, 2020).

\section{REFERENCIAS BIBLIOGRÁFICAS}

Antonio-Aguirre, I., Rodríguez-Fernández, A. y Revuelta, L. (2019). El impacto del apoyo social y la inteligencia emocional percibida sobre el rendimiento académico en Educación Secundaria. European Journal of Investigation in Health, Psychology and Education, 9(2), 109-118.

Connor, K. M., y Davidson, J. R. (2003). Development of a new resilience scale: The Connor-Davidson resilience scale (CD-RISC). Depression and Anxiety, 18(2), 76-82. doi:10.1002/da.10113

Diamond, A. (2013). Executive Functions. Annual Review of Psychology, 64, 135-168.

Escolano-Pérez, E. y Bestué, M. (2021) Academic Achievement in Spanish Secondary School Students: The InterRelated Role of Executive Functions, Physical Activity and Gender. International Journal of Environmental Research and Public Health, 18(4):1816. https://doi.org/10.3390/ijerph18041816

Fínez-Silva, M. J., Morán-Astorga, C., y Urchaga-Litago, J. D. (2019). Resiliencia psicológica a través de la edad y el sexo. International Journal of Developmental and Educational Psychology, 4(1), 85-94. https://www.redalyc.org/articulo.oa? id=349861666009

García, M. Á., González-Gómez, A., Robles-Ortega, H., Padilla, J. L., y Peralta- Ramirez, I. (2019). Propiedades psicométricas de la Escala de Resiliencia de Connor y Davidson (CD-RISC) en población española. Anales de Psicología/ Annals of Psychology, 35(1), 33-40.

Gioia, G.A., Isquith, P.Q., Guy, S.C., Kenworthy, L., Roca, M.E.S. y Santamaría, P. (2017) BRIEF-2: Evaluación Conductual de La Función Ejecutiva 2; TEA: Madrid, Spain.

Goldberg, E. (2009). The new executive brain: Frontal lobes in a complex world. Oxford University Press.

Grissom, N. M., y Reyes, T. M. (2019). Let's call the whole thing off: evaluating gender and sex differences in executive function. Neuropsychopharmacology, 44(1), 86-96.

Ley Orgánica 3/2020 (LOMCE), de 29 de diciembre, por la que se modifica la Ley Orgánica 2/2006 del 3 de mayo, de Educación. BOA 30 de diciembre de 2020. Disponible en: https://www.boe.es/eli/es/lo/2020/12/29/3

Lezak, M. D. (1982). The problem of assesing executive functions. International Journal of Psychology, 17(2), 281-297.

Lezak, M. D., Howieson, D.B. y Loring D.W. (2004). Neuropsychological assessment. Nueva York: Oxford University Press. 
Matthews, J. S., Ponitz, C. C., y Morrison, F. J. (2009). Early gender differences in self- regulation and academic achievement. Journal of educational psychology, 101(3), 689. https://doi.org/10.1037/a0014240

Ministerio de Educación y Formación Profesional (2021). Neurociencia aplicada a la educación. Disponible en: https://www.educacionyfp.gob.es/va/mc/neurociencia- educativa/recursos/recursos-fejecutivas-patencionales/programas- intervencion.html

Miñano, P. y Castejón, J. L. (2011). Variables cognitivas y motivacionales en el rendimiento académico en Lengua y Matemáticas: un modelo estructural. Revista de Psicodidáctica, 16(2),203-230. https://www.redalyc.org/articul0.0a? id=17518828003

Miyake, A., y Friedman, N. P. (2012). The nature and organization of individual differences in executive functions: Four general conclusions. Current Directions in Psychological Science, 21(1), 8-14

Organisation for Economic Co-operation and Development (OECD). (2020). Panorama de la educación. Indicadores de la OCDE 2020. Disponible en https:// www.educacionyfp.gob.es/inee/indicadores/indicadores-internacionales/ ocde.html

Sánchez-Álvarez, N., Berrios Martos, M. P., y Extremera, N. (2020). A meta-analysis of the relationship between emotional intelligence and academic performance in secondary education: A multi-stream comparison. Frontiers in psychology, 11, 1517.

Sastre-Riba, S. (2006). Condiciones tempranas del desarrollo y el aprendizaje: el papel de las funciones ejecutivas. Revista de Neurología, 42(Supl 2), 143-151.

Sun, J., y Stewart, D. (2007). Age and gender effects on resilience in children and adolescents. International Journal of mental health promotion, 9(4), 16-25. https://doi.org/10.1080/14623730.2007.9721845

Vinaccia, S., Quiceno, J. M., y Moreno, E. (2007). Resiliencia en adolescentes. Revista colombiana de psicología, 16, 139-146.

Wu, L., Zhang, X., Wang, J., Sun, J., Mao, F., Han, J. y Cao, F. (2021). The associations of executive functions with resilience in early adulthood: A prospective longitudinal study. Journal of Affective Disorders, 282, 1048-1054.

Zelazo, P. D. y Carlson, S. M. (2020). The neurodevelopment of executive function skills: Implications for academic achievement gaps. Psychology \& Neuroscience, 13(3), 273.

Zhang, Y., Zhang, X., Zhang, L. y Guo, C. (2019). Executive function and resilience as mediators of adolescents' perceived stressful life events and school adjustment. Frontiers in psychology, 10, 446. 